\title{
Anisotropy in the Australasian upper mantle from Love and Rayleigh waveform inversion
}

\author{
Eric Debayle ${ }^{\mathrm{a}, \mathrm{b}, *}$, B.L.N. Kennett ${ }^{\mathrm{a}}$ \\ a Research School of Earth Sciences, the Australian National University, Canberra, A.C.T. 0200, Australia \\ b Ecole et Observatoire des Sciences de la Terre, CNRS and Université Louis Pasteur, 5 rue René Descartes, 67084 Strasbourg, France
}

Received 11 July 2000; received in revised form 16 October 2000; accepted 16 October 2000

\begin{abstract}
Records of both Rayleigh and Love waves have been analyzed to determine the pattern of anisotropy in the Australasian region. The approach is based on a two-stage inversion. Starting from a smooth PREM model with transverse isotropy about a vertical symmetry axis, the first step is an inversion of the waveforms of surface waves to produce path specific one-dimensional (1-D) upper mantle models. Under the assumption that the 1-D models represent averages along the paths, the results from 1584 Love and Rayleigh wave seismograms are combined in a tomographic inversion to provide a representation of three-dimensional structure for wavespeed heterogeneities and anisotropy. Polarization anisotropy with SH faster than SV is retrieved in the upper 200-250 km of the mantle for most of Precambrian Australia. In this depth interval, significant lateral variations in the level of polarization anisotropy are present. Locally, the anisotropy can be large, reaching an extreme value of $9 \%$ that is difficult to reconcile with current mineralogical models. However, the discrepancy may be explained in part by the presence of strong lateral heterogeneities along the path, or by effects introduced by the simplifying assumption of transverse isotropy for each path. The consistency between the location of polarization and azimuthal anisotropy in depth suggests that both observations share a common origin. The observation of polarization anisotropy down to at least $200 \mathrm{~km}$ supports a two-layered anisotropic model as constrained by the azimuthal anisotropy of SV waves. In the upper layer, $150 \mathrm{~km}$ thick, anisotropy would be related to past deformation frozen in the lithosphere while in the lower layer, anisotropy would reflect present day deformation due to plate motion. (C) 2000 Elsevier Science B.V. All rights reserved.
\end{abstract}

Keywords: anisotropy; Australia; surface waves; upper mantle; tomography

\section{Introduction}

Although upper mantle anisotropy has been observed in many parts of the world since the $60 \mathrm{~s}[1,2]$, the relative role of seismic heterogeneities and anisotropy remains a difficult issue to

\footnotetext{
* Corresponding author. E-mail: eric@sismo.u-strasbg.fr
}

address with current seismological techniques. Petrophysical studies show that the lattice preferred orientation of anisotropic minerals should produce S-wave anisotropy of a few percent in the upper mantle [3], a result compatible with most SKS and surface waves studies.

Surface wave data can be used to constrain anisotropic directions. For Rayleigh waves, a relatively modest number of crossing paths is necessary because the azimuthal variation is dominated 
by a term that depends on $\cos 2 \theta, \sin 2 \theta$ where $\theta$ is the azimuth (e.g. [4]). However, the amplitude of azimuthal anisotropy still remains difficult to estimate accurately. As an example, since many isotropic tomographic upper mantle models have been found to give reasonable fit to Rayleigh wave dispersion data, it can be acceptable to suppress azimuthal anisotropy.

The incompatibility between isotropic shear velocity models derived from Love and Rayleigh waves is generally considered as a stronger diagnostic for the presence of anisotropy in the upper mantle. This class of anisotropy is commonly referred to as polarization or radial anisotropy. At a global scale, polarization anisotropy tomographic models seem to image down- and upgoing flow in the mantle [5,6]. At regional scale, few polarization anisotropy models are available for continents. However, for Australia, body and surface waves have been used to build a one-dimensional (1-D) polarization anisotropy model for a single corridor crossing the Precambrian part of the continent [7].

In this paper, we investigate lateral variation in both the polarization and azimuthal anisotropy beneath the Australasian region. For this purpose, we use Love and Rayleigh wave seismograms recorded at the permanent IRIS and GEOSCOPE stations in the Australian region, supplemented with data recorded at the portable broad-band stations deployed in Australia in the SKIPPY experiment [8] and a subsequent deployment of eight broad-band stations in the Kimberley, West Australia from July to October 1997 (KIMBA experiment). Polarization anisotropy is obtained from 792 paths by the waveform inversion of both Love and Rayleigh seismograms (i.e. 1584 waveforms). Azimuthal anisotropy is constrained from the azimuthal variation of the Rayleigh waves.

\section{Tomographic procedure}

The tomographic procedure used in this study is a two-stage process. The first stage is a waveform inversion [9]. For each epicenter station path, an average 1-D upper mantle model that simultaneously represents the waveforms of both Love and Rayleigh waves is found using synthetics calculated for the fundamental and first few higher modes for each wavetype $[10,11]$ in a transversely isotropic model.

In the second stage, a continuous regionalization algorithm [12] is applied to the path-averaged 1-D models in order to retrieve the local threedimensional (3-D) structure, for both SV and SH wavespeeds.

\subsection{Dataset}

The tomographic images presented in this paper are constrained by 1584 Love and Rayleigh wave seismograms, each Love and Rayleigh pair corresponding to a single event. On the 792 Rayleigh wave seismograms, 451 were included in a previous inversion of a larger Rayleigh wave dataset by [13]. Because for a given frequency the Love wave fundamental mode has a depth penetration shallower than Rayleigh, we invert the waveforms in the period range $50-160 \mathrm{~s}$ instead of $40-160 \mathrm{~s}$ in the previous Rayleigh only inversion to limit possible artifacts due to variations in crustal structure. The data have been selected using the signal to noise ratio criterion defined in [11] but applied to the seismograms filtered around the central periods $50,70,120 \mathrm{~s}$ and $50,90,160 \mathrm{~s}$.

The waveform inversion is then performed for the fundamental mode and up to four overtones of the Love and Rayleigh waveforms in the period range 50-160 s, depending on the signal to noise ratio available for each mode at each period. Higher modes have been considered in the inversion for 505 Rayleigh wave and 785 Love wave seismograms. In the period range of analysis, higher modes provide good resolution at least down to $400 \mathrm{~km}$ depth.

\subsection{Parameterization}

The 1-D upper mantle models obtained for each path of this study allow the $\mathrm{S}$ velocities deduced from Rayleigh and Love waves to propagate at different wavespeeds and thus contain an information on polarization anisotropy. This information is mixed together with an azimuthal 
component also present in the 1-D path average upper mantle models ([14], equation 3). There are several ways to deal with the azimuthal component when combining the path average models in the tomographic inversion, and this issue is discussed in Section 2.4 of this paper.

However, the azimuthal component is not included in the waveform inversion for individual 1D upper mantle models. This is probably an oversimplification, since an azimuthal dependence is expected from the preferred orientation of anisotropic minerals with fast axis close to the horizontal, a situation likely to be common in the upper mantle. A number of studies suggest that such polarization anisotropy measurements beneath individual paths, without corrections for the azimuthal component are still useful [7,15]. Synthetic dispersion curves have been computed about a fixed horizontal axis in a medium with hexagonal symmetry that mimic the preferred orientation of anisotropic mineral [15]. With an inversion assuming a transversely isotropic model about a vertical symmetry axis (TIV), polarization anisotropy is retrieved at the right depth but with a distorted amplitude. At low frequencies, stochastic models of upper mantle heterogeneity in which orthorombic peridotites have fast axes that are preferentially horizontal but randomly oriented in azimuth yield TIV effective media [7]. We would therefore expect to retrieve at least the depth location of anisotropy from our set of path average 1-D upper mantle models.

In a TIV medium, surface waves are sensitive to five elastic parameters, the velocities of vertical and horizontal propagation for $\mathrm{P}\left(\alpha_{v}, \alpha_{h}\right)$ and $\mathrm{S}$ $\left(\beta_{v}, \beta_{h}\right)$ waves and a parameter $\eta$ related to propagation at intermediate angles [16], as well as the quality factor $Q_{\beta}$ and the density $\rho$. Surface wave information is not sufficient to resolve all the parameters (see e.g. [17]). We have therefore restricted attention in the inversion to the best resolved parameters, the SV velocity $\beta_{v}$, the S-wave anisotropic parameter $\xi=\left(\beta_{h} / \beta_{v}\right)^{2}$, and the quality factor parameterized as $\log Q_{\beta}$. The scalar seismic moment of the source $\log \left(M_{0}\right)$ is added to ensure an adequate scaling of the signal. The inversion is performed for upper mantle structure only. For each path, an average crustal structure along the path is constructed from the 3-D a priori model 3SMAC [18]. We also use the local structure of the 3SMAC model taken in the source region to compute the source excitation.

\subsection{Model construction}

Polarization anisotropy has been locally observed in the upper mantle of northern Australia [19] and beneath a NE-SW seismic corridor from Papua New-Guinea to Western Australia [7]. To check if these findings could be extended to the whole continent, we first tried to match our set of Love and Rayleigh wave seismograms with an isotropic inversion. We used PREM as a starting model, but removed the anisotropy present in the top $220 \mathrm{~km}$ of the upper mantle and inverted only for isotropic parameters. Satisfactory waveform matching could be achieved for 358 paths, but few of them cross continental Australia (Fig. 1a). In addition, a poor variance reduction was obtained after the regionalization of the path average models, suggesting that anisotropy had been mapped into the individual 1-D models through artificial perturbations of the path average isotropic parameters.

We therefore extended the inversion to include radial anisotropy in the model. The starting model was a smoothed version of PREM, and waveform matching was achieved for 1584 Love and Rayleigh seismograms. The path coverage is displayed in Fig. 1b.

We have extracted the SH velocity $\beta_{h}$ from the path average $\xi$ models, and then used both the $\mathrm{S}$ velocities in calculating the tomographic results presented in Figs. 2 and 3. The regionalization algorithm is based on the path average approximation that the propagation time is the integral of the appropriate slowness along the great circle path. The $\xi$ parameter has been recomputed for Fig. 2 while in Fig. 3 the absolute $\beta_{h}$ and $\beta_{v}$ velocities are directly represented, the bars display the azimuthal anisotropy for $\beta_{v}$.

\subsection{Retrieving anisotropy}

In terms of the azimuth of propagation $\theta$, the expected azimuthal variation for $\mathrm{SV}$ waves 

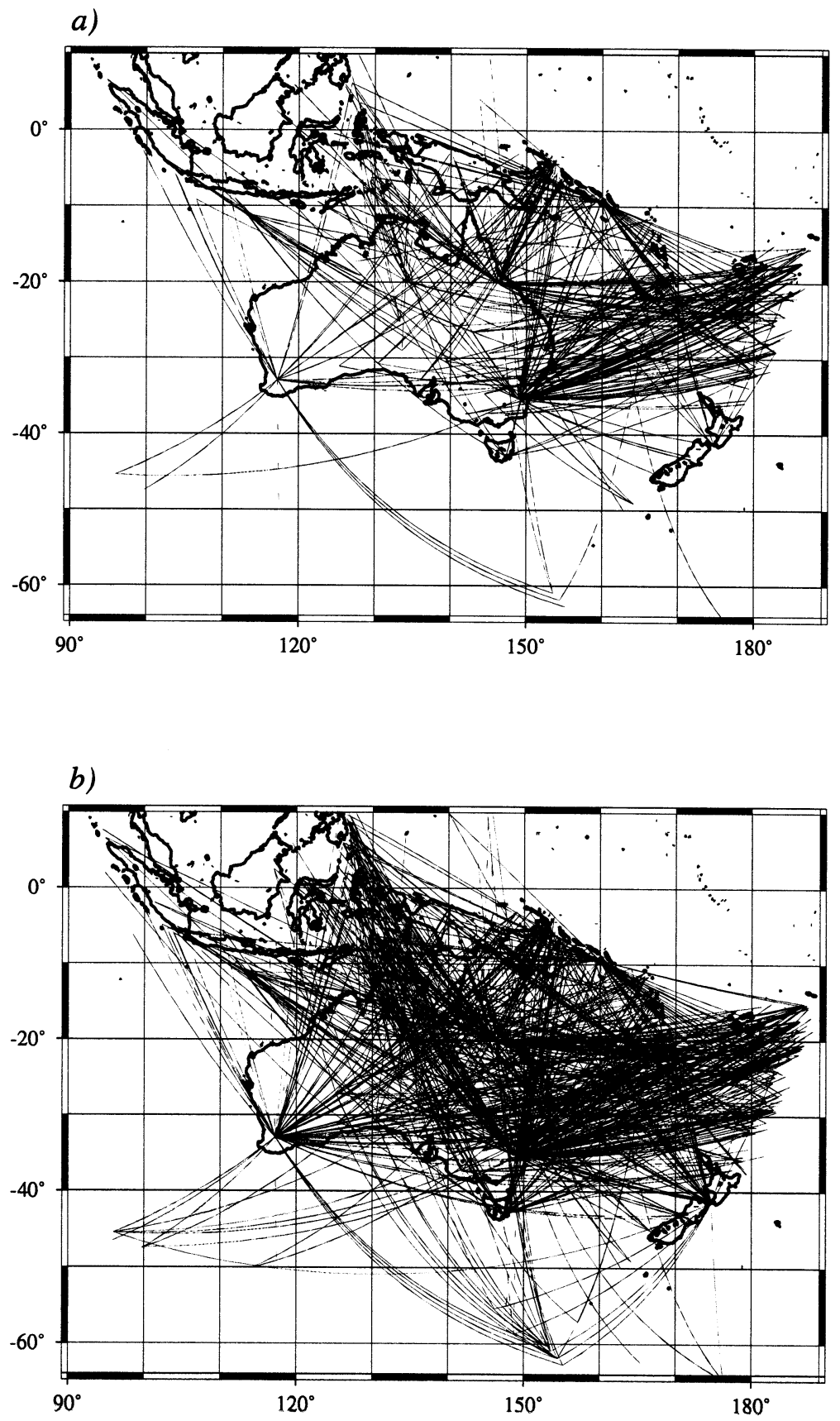

Fig. 1. Path coverage achieved for simultaneous Rayleigh and Love waveform inversion. (a) With no anisotropy allowed in the inversion and no anisotropy in the starting model (the starting model is a smooth PREM with the anisotropy removed). (b) Allowing polarization anisotropy to be present in the inverted model and starting from a smooth PREM. 

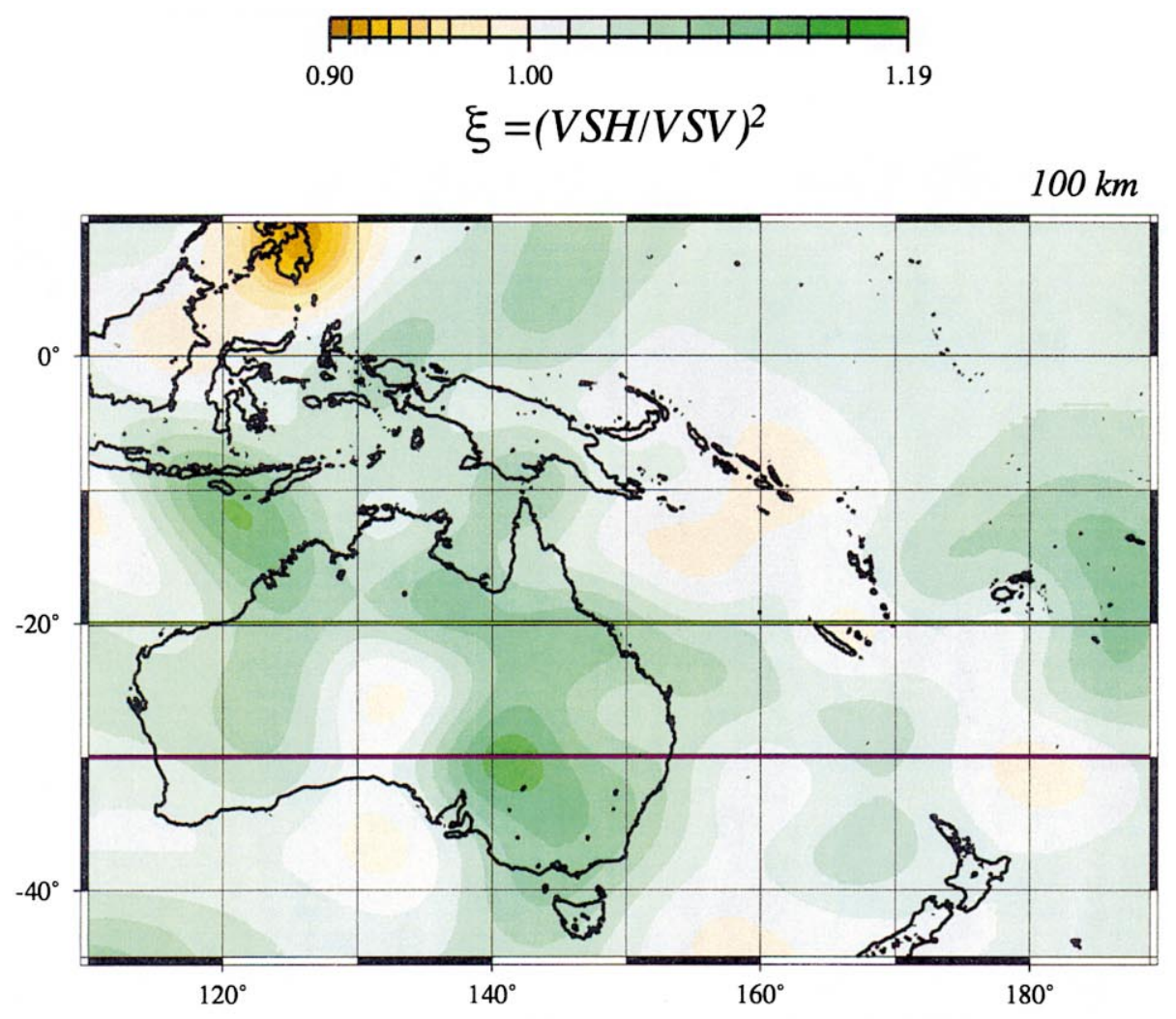

$20^{\circ} \mathrm{S}$
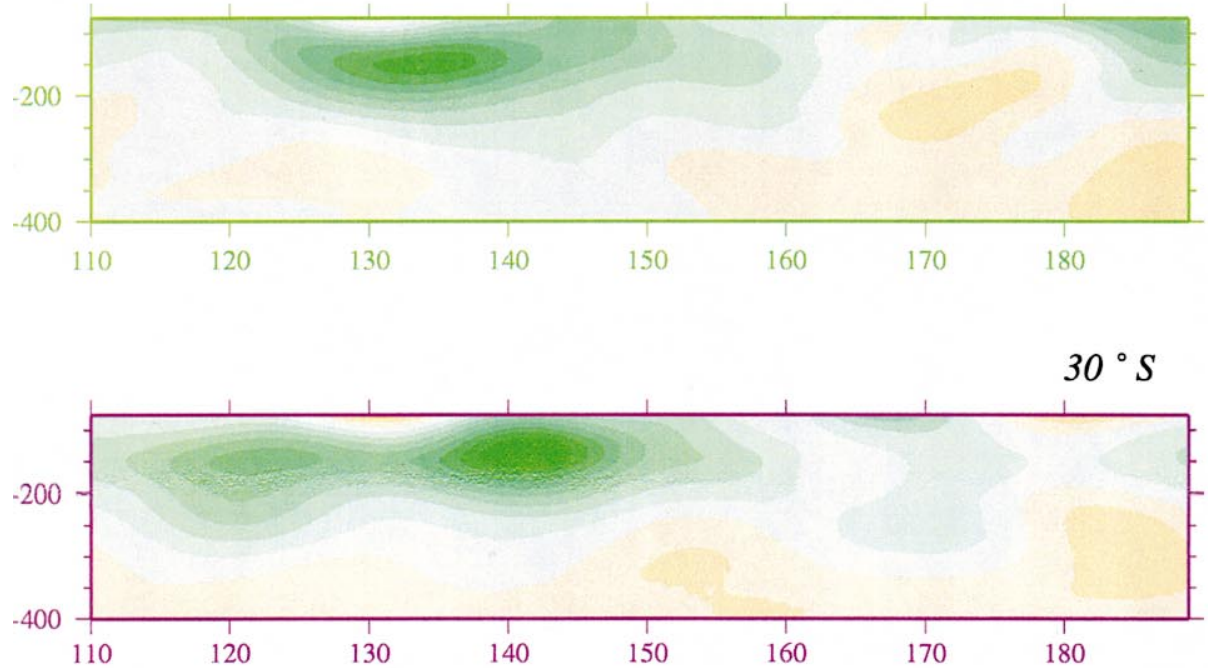

Fig. 2. Polarization anisotropy model at $125 \mathrm{~km}$ depth with two vertical cross-sections at $-20^{\circ} \mathrm{S}$ and $-30^{\circ} \mathrm{S}$. SH waves propagate significantly faster than SV waves in the uppermost $200-250 \mathrm{~km}$ for most of Australia. 

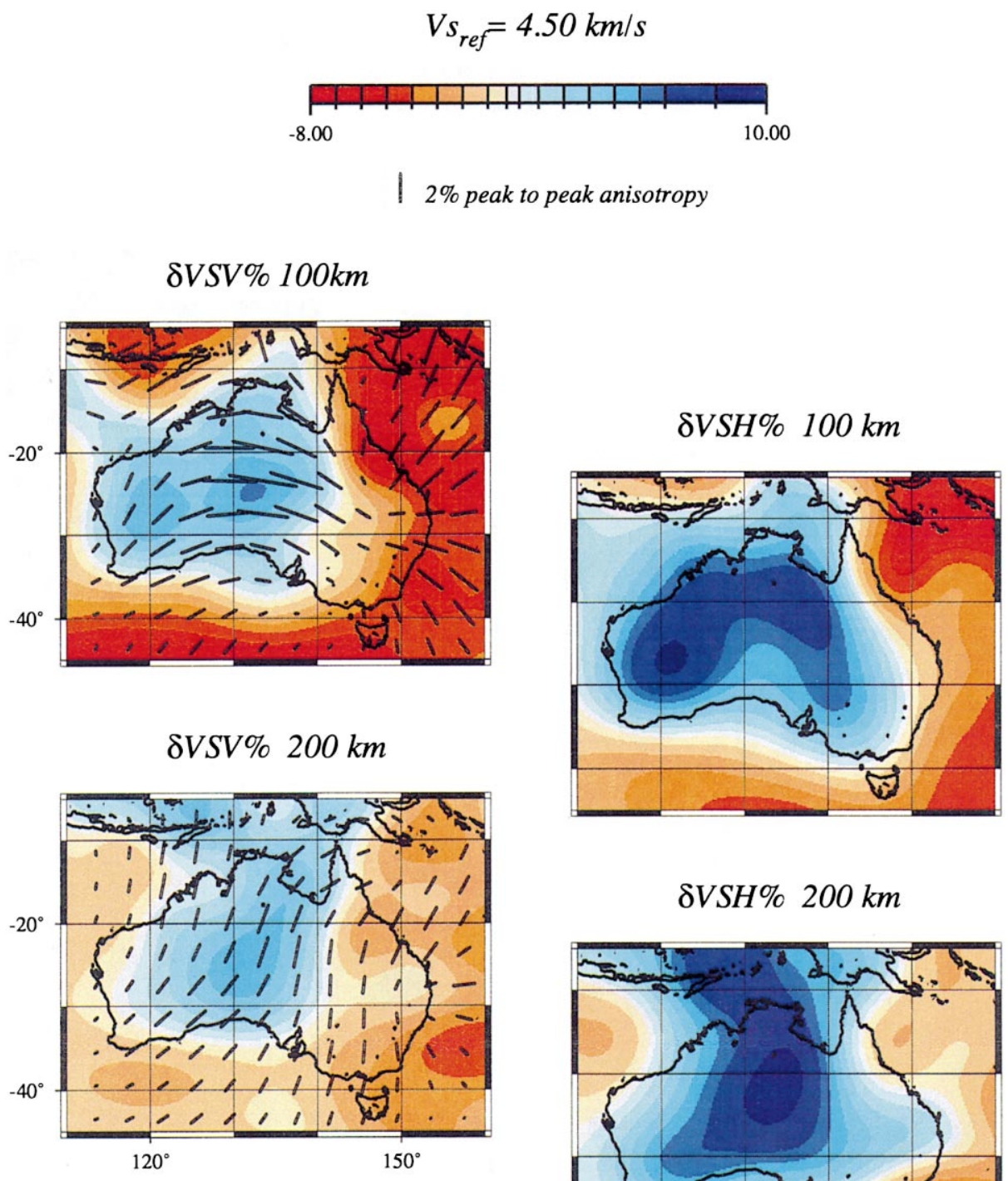

\section{$\delta V S H \% 200 \mathrm{~km}$}

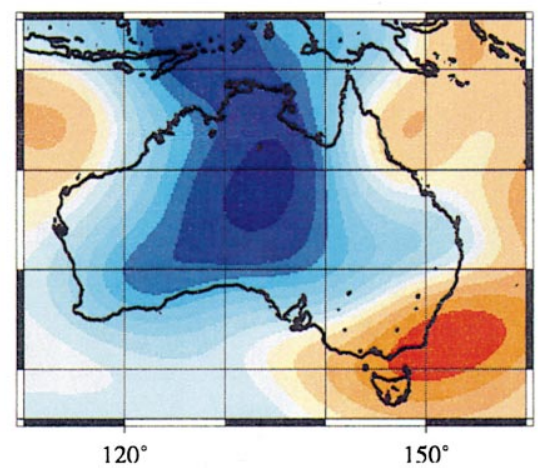

Fig. 3. Left column: SV wave heterogeneities and azimuthal anisotropy; right column: SH wave heterogeneities (tones represent velocity contrasts and bars display azimuthal anisotropy, the length of the bars indicating the strength of anisotropy).

(mainly associated with Rayleigh waves) depends on $\cos 2 \theta$, sin $2 \theta$ and can readily be distinguished from the isotropic component. We have therefore chosen to separate the isotropic and anisotropic components in the $\beta_{v}$ regionalization. For $\mathrm{SH}$ waves (mainly constrained by the Love waves) the expected azimuthal variation depends on cos
$4 \theta, \sin 4 \theta$ and needs a much better path coverage to be resolved (see e.g. [14]). In addition, a faster azimuthal variation should be averaged out more easily by the propagation paths. This is important because if the propagation paths are sufficient to average over the azimuthal dependency, the inverted velocities should be representative of the 


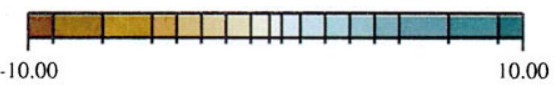

$$
\delta V s(\%) \quad V s_{r e f}=4.5 \mathrm{~km} / \mathrm{s}
$$

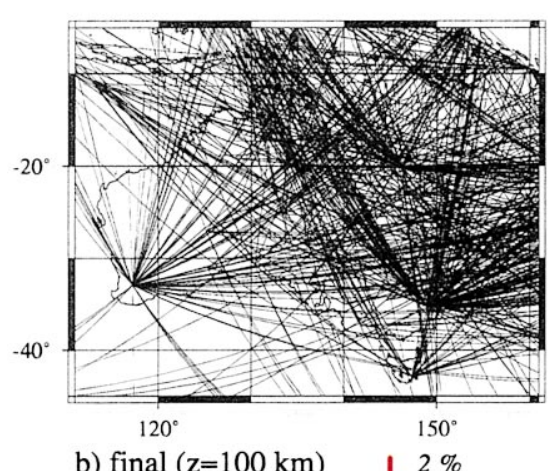

b) final $(\mathrm{z}=100 \mathrm{~km}) \quad$ | $2 \%$

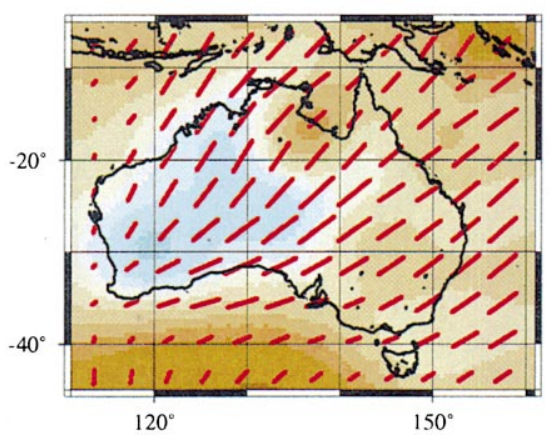

a) initial

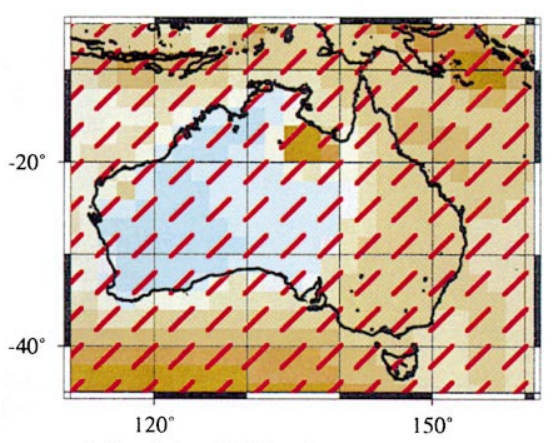

c) final $(z=200 \mathrm{~km})$

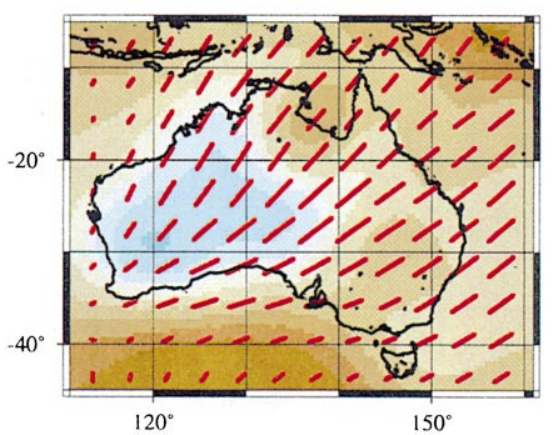

Fig. 4. Synthetic experiment: (left, top) actual path coverage used for the synthetic test. (a) The initial model is the isotropic shear velocity distribution at $50 \mathrm{~km}$ depth provided by the 3SMAC model of [18] with a pattern of azimuthal anisotropy superimposed. (b) Result of the synthetic inversion at $100 \mathrm{~km}$ depth. (c) Result of the synthetic inversion at $200 \mathrm{~km}$ depth.

average of the true velocities [20]. In the tomographic inversion we aim when considering the path coverage to choose a degree of smoothing that allows us either to solve for the azimuthal variation, or to average it out if the azimuthal distribution of rays is not sufficient.

After several trials, we chose a value of $L_{\text {corr }}=500 \mathrm{~km}$ for the standard deviation of the a priori Gaussian function that controls the lateral smoothness of the model in the continuous regionalization algorithm [12]. With our path coverage, this is sufficient to separate the azimuthal variation of $\beta_{v}$ from the isotropic component. To test this intuitive idea, we have performed various synthetic experiments where we try to re- trieve a shear velocity distribution in the mantle with a given pattern of anisotropy superimposed. We present in Fig. 4 the results obtained for an initial model made by superimposing $2 \%$ anisotropy with azimuth $45^{\circ} \mathrm{N}$ on the $\mathrm{S}$ velocity provided by the 3SMAC model [18] at a depth of $50 \mathrm{~km}$. The red bars correspond to the directions of fast $\beta_{v}$ propagation extracted from the $2 \theta$ variation. Seismic heterogeneities are smoothed out by the $500 \mathrm{~km}$ value of $L_{\text {corr }}$ but east of $130^{\circ} \mathrm{E}$, both the anisotropic directions and the seismic heterogeneities are very well retrieved. Similar results have been obtained for synthetic tests with other anisotropic directions. The observation that we can determine the $\cos 2 \theta, \sin 2 \theta \mathrm{SV}$ anisotropic direc- 
tions with our a priori choices means that east of $130^{\circ} \mathrm{E}$, we sample each cell of $500 \mathrm{~km}$ with a minimum of three different azimuths. The path coverage of Fig. $1 \mathrm{~b}$ suggests that most $500 \mathrm{~km}$ cells located east of $130^{\circ} \mathrm{E}$ are in fact sampled by more than three different azimuths. However, our azimuthal coverage is probably not sufficient to solve the $4 \theta$ azimuthal variation of $\beta_{h}$ which would require each $500 \mathrm{~km}$ cell to be sampled by a minimum of five different azimuths. In most situations, a minimum of three different azimuths should provide a good average of the $\mathrm{SH}$ wave azimuthal variation in $\cos 4 \theta, \sin 4 \theta$ so that the inverted $\mathrm{SH}$ velocities should be close to the isotropic component of the velocity distribution.

The differences between $\beta_{h}$ and the isotropic component of $\beta_{v}$ thus provide information on the polarization anisotropy. The directions of fast $\beta_{v}$ propagation extracted from the $2 \theta$ variation and represented by the black bars of Fig. 3 should correspond to the fast $a$-axis of olivine crystals in an upper mantle mineralogy dominated by olivine (see e.g. [14] for a more complete discussion on this point).

\section{A posteriori error and resolution}

We present in Figs. 5 and 6 slices through the a posteriori error models computed for the $\xi, \beta_{v}$ and $\beta_{h}$ parameters. The dark gray areas correspond to part of the models where the a posteriori error is close to the a priori error, indicating a total lack of resolution. In Figs. 5 and 6, these areas are restricted to the border of the maps and most of the Australian continent is associated with small errors indicating good resolution. A NE-SW gradient is present in the error maps, and the best resolved areas are located to the northeast of Australia. We note from Fig. 6 a slight increase in the a posteriori error between 100 and $200 \mathrm{~km}$ depth. At larger depths, higher modes provide good resolution down to $400 \mathrm{~km}$ depth and on Fig. 5, the a posteriori error remains relatively constant in the vertical cross-sections. The presence of polarization anisotropy cannot be considered as significant when the $\xi$ parameter differs from 1 by less than the a poste- riori error. Comparing Figs. 5 and 2 we would argue that significant polarization is present only down to $200-250 \mathrm{~km}$ in the upper mantle. Below this depth, if anisotropy is present, it is probably weak and below the resolution of our data.

\section{Results and discussion}

If flow in the mantle is mainly horizontal, then evidence from fabric of peridotite nodules suggests that the average $\beta_{h}$ would be larger than the average $\beta_{v}$, resulting in $\xi$ values larger than 1 (e.g. [21]). In this study, polarization anisotropy is well resolved east of $130^{\circ} \mathrm{E}$, where our path coverage is sufficient to average out the azimuthal variation of $\mathrm{S}$ waves and thus solve for the $\xi$ parameter. We are therefore able to discuss the anisotropy in the central Proterozoic part of the continent and in the eastern third of Australia, generally associated with Paleozoic basement. Significant variations occur in the amplitude of polarization anisotropy throughout Australia but for most of the continent $\beta_{h}$ is larger than $\beta_{v}$ in the uppermost $200-250 \mathrm{~km}$ of the mantle, while deeper, anisotropy is weak (Fig. 2). Regions where the $\xi$ parameter falls below unity are observed in the superficial layer of Central Australia, in the Cape York region and in most of the subduction zones surrounding the continent, where vertical flow dominates.

The $\beta_{h}$ and $\beta_{v}$ heterogeneity maps of Fig. 3 present somewhat different patterns. On the $\beta_{v}$ map at $100 \mathrm{~km}$ depth, the western two-thirds of the Australian continent, underlain by Precambrian crust, display high seismic wavespeeds while the eastern Phanerozoic remainder shows lower seismic velocities. In the $\beta_{h}$ map at $100 \mathrm{~km}$, the fast velocities do not outline the shape of the Proterozoic craton but extend further east under $\mathrm{Pa}$ leozoic Australia and this difference persists down to $200 \mathrm{~km}$, although it is less pronounced at greater depth. This region between $138^{\circ} \mathrm{E}$ and $148^{\circ} \mathrm{E}$, which marks the transition from the Precambrian craton to the Phanerozoic terranes is characterized by a large polarization anisotropy in the uppermost $200 \mathrm{~km}$ (Fig. 2) of the mantle. A closer correspondence in the geographic pattern of 

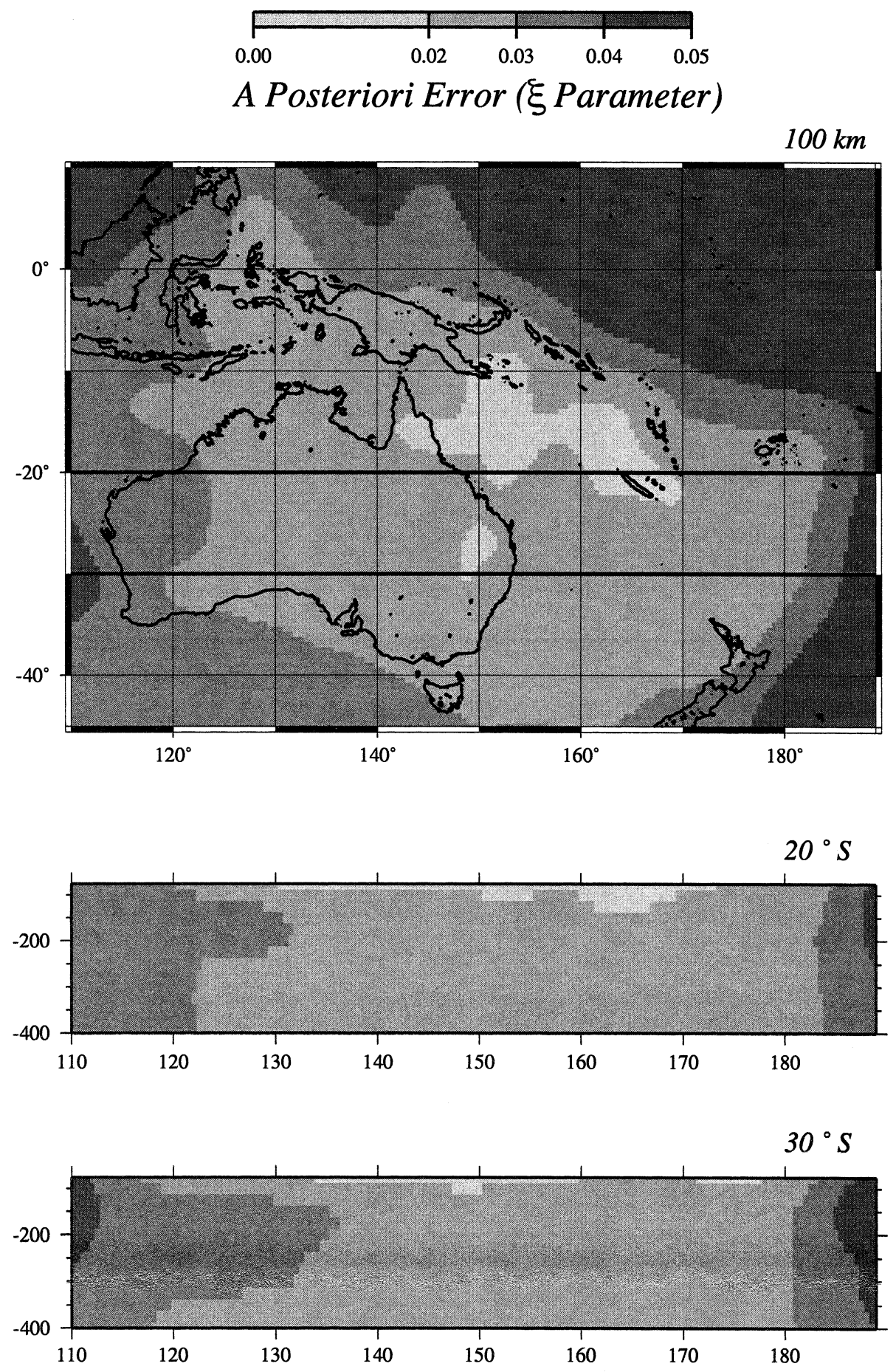

Fig. 5. A posteriori error distribution for the $\xi$ parameter presented in Fig. 2. 


\section{A Posteriori Error $(\mathrm{km} / \mathrm{s})$}
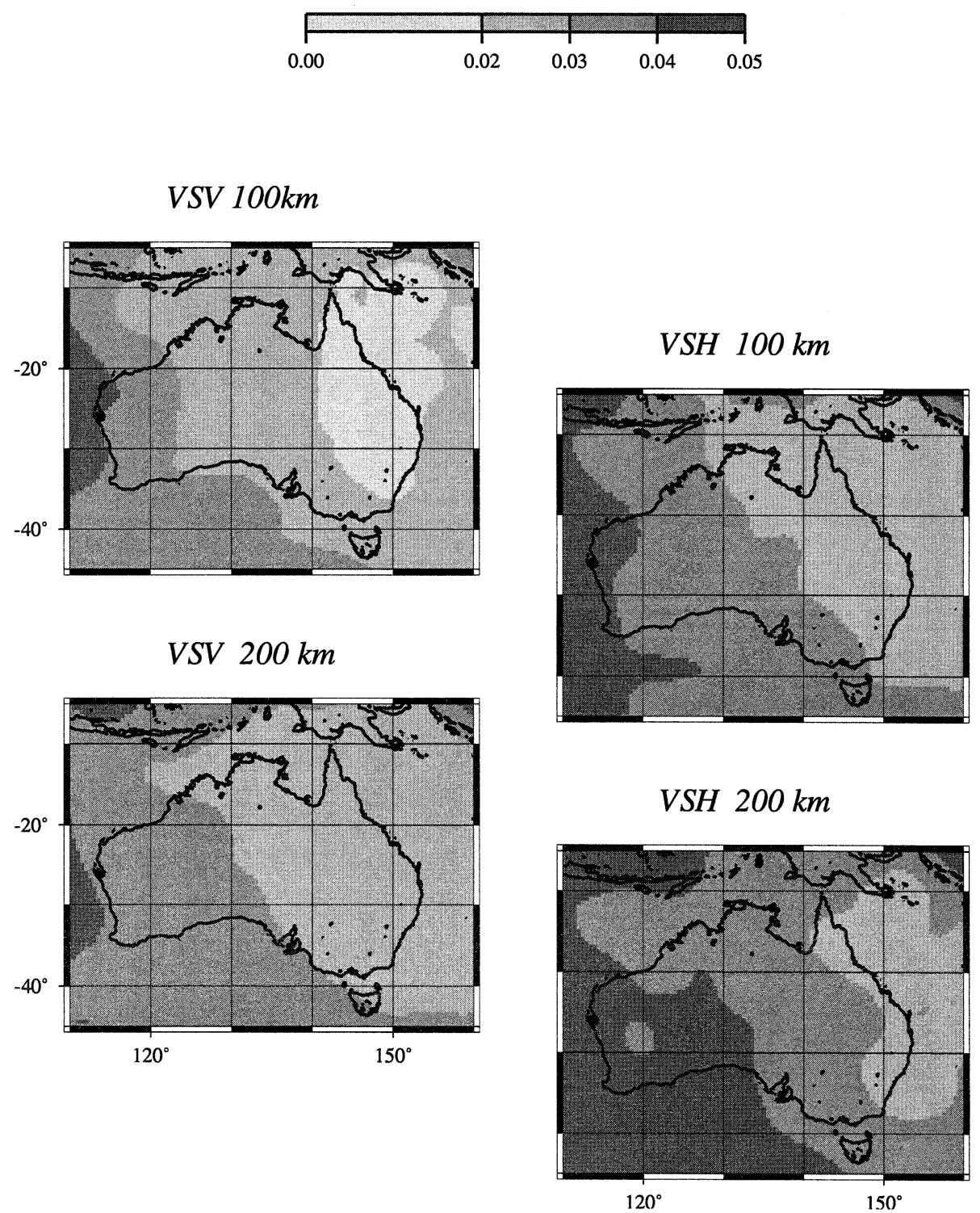

Fig. 6. A posteriori error distribution for the $\mathrm{SV}$ and $\mathrm{SH}$ wave heterogeneities presented in Fig. 3.

anomalies would be obtained with a $5 \%$ increase in the reference velocity for $\beta_{h}$ used for plotting the distribution.

Azimuthal anisotropy is also present in the uppermost $200-250 \mathrm{~km}$ for $\beta_{v}$ but is organized in two layers (Fig. 3). The origin of these two layers has been discussed in [13] from a dataset of 2194 Rayleigh wave seismograms. The upper layer is likely to be related to deformation frozen in the lithosphere, while the smoother pattern with dominant north-south component in the lower layer would suggest present day deformation due to shearing of the mantle by the plate motion. In Fig. 3, present day deformation occurs 
at $200 \mathrm{~km}$ depth within the region of high seismic velocities. The depth at which present day deformation occurs in the mantle is likely to reflect the thickness of the 'mechanical' lithosphere, the part of the mantle that presents coherent horizontal motion. This 'mechanical' lithosphere may differ from the 'seismic' lithosphere determined from the thickness of the high velocity lid and more likely reflects thermal and compositional (via depletion of the cratonic keel) effects. As an example, seismic anisotropy is probably mostly sensitive to the orientation of olivine crystals in the mantle. According to current geotherms for Precambrian cratons a temperature of $1100-1200 \mathrm{~K}$ is reached just above $150 \mathrm{~km}$ depth (i.e. [22]). This would correspond to a threshold temperature at which the mobility of olivine crystals is sharply enhanced [20]. If correct, frozen deformation may not be preserved below $150 \mathrm{~km}$ depth as it would be erased by more recent deformation. This scenario is in good agreement with our results. In our model, the transition between the two layers is marked by a change in the pattern of anisotropic directions at around $150 \mathrm{~km}$ depth, within the $200-250 \mathrm{~km}$ thick layer where significant and consistent polarization anisotropy is observed for Precambrian Australia.

Mapping the anisotropic directions as a function of depth provides an essential clue to distinguish the changes in the organization of anisotropy and hence its origin. Polarization anisotropy provides us with independent evidence for the presence and depth extent of the anisotropic layers. Our results agree with the previous observation of polarization anisotropy in the uppermost $250 \mathrm{~km}$ of the mantle beneath Precambrian Australia [7]. We however observe locally a larger polarization anisotropy, that reaches $9 \%$ at 150 $\mathrm{km}$ depth in eastern Australia. This is an extreme value, that cannot be reconciled with current mineralogical models. An olivine model with all crystals oriented in the horizontal plane would produce $8 \%$ of polarization anisotropy assuming a pyrolitic model [23]. We have checked this result by using a set of different starting models; similar results were obtained with a PREM model with the upper mantle anisotropy removed or the AU3 model [7]. We also tried to invert the Love and
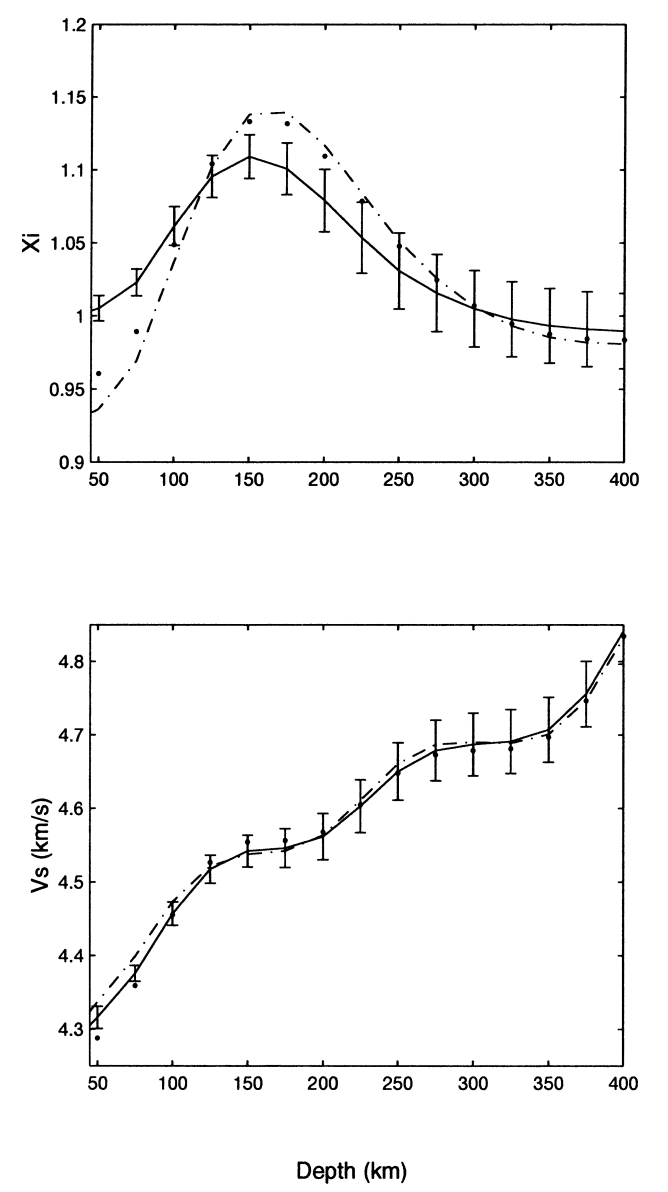

Fig. 7. Example of the effect produced by a $10 \mathrm{~km}$ variation in crustal thickness on a typical path average SV velocity (bottom) and polarization anisotropy (top) model. The 1-D elastic models have been obtained after the analysis of the Love and Rayleigh waveforms recorded at the Canberra GEOSCOPE station after the 8 November 1995 earthquake which occurred near the Celebes Island at lat $=1.32^{\circ} \mathrm{N}$ and lon $=121.72^{\circ} \mathrm{E}$. The model obtained assuming a crustal thickness of $32 \mathrm{~km}$ after averaging the 3SMAC model [18] along the path is displayed with its error bars using solid lines. Inverted models are also shown after changing the crustal thickness to $27 \mathrm{~km}$ (dotted line) and $37 \mathrm{~km}$ (dashdot line).

Rayleigh seismograms separately. We always ended up with differences between $\beta_{v}$ and $\beta_{h}$ of the same order.

Several factors may contribute to explain these large differences between SV and SH wavespeeds. In this study, we are looking at local anisotropic structure while previous polarization anisotropy observations for Australia are average estima- 
tions, either along an epicenter station seismic corridor [7] or for the northern part of the continent [19]. There is also likely to be some tradeoff between strong lateral heterogeneity along the paths and polarization anisotropy ([24,25]) and this may contribute to the large anisotropy observed in eastern Australia where strong contrasts are present between the craton and the Phanerozoic basement. Crustal contamination may also create artificial anisotropic signal. We present in Fig. 7 the effect produced by a $10 \mathrm{~km}$ variation in crustal thickness on one of our path average models. In this study, the typical path average thickness would be about $30 \mathrm{~km}$ because most of the paths cross part of the roughly $40 \mathrm{~km}$ thick crust but also include a significant proportion of oceanic areas. We choose a path from the Celebes Island to Canberra with a crustal thickness of 32 $\mathrm{km}$ obtained after averaging the 3SMAC model along the path. The effect of changing the crust to 27 and $37 \mathrm{~km}$ is shown for the path average models in Fig. 7. The deviation exceeds the error bars only in the uppermost $100 \mathrm{~km}$ for the shear velocity. For polarization anisotropy the effects are more pronounced especially at shallowest depths, but the depth location of polarization anisotropy is not affected. The example presented in Fig. 7 is likely to represent an extreme upper bound for crustal effects. A $200 \mathrm{~km}$ wide zone with a 10 $\mathrm{km}$ difference in crustal thickness will only produce a difference in $2 \mathrm{~km}$ in the average crustal model for paths as short as $2000 \mathrm{~km}$. However, the necessity of working with paths with mixed continental and oceanic components means that there is inevitably some influence from crustal structure.

Generally only crustal errors averaged along the epicenter station path have to be considered and the resulting velocity perturbation has the same sign on both the Love and Rayleigh fundamental mode phase velocities. For a given period, it is therefore only the difference in the Love and Rayleigh wave sensitivity to the crustal errors averaged along the path that has to be considered. Keeping in mind that the shortest period used in this study is $50 \mathrm{~s}$, we do not expect crustal effects to play a major role in the amplitude of the discrepancy. It seems to us more likely that the sim- plifying assumption of transverse isotropy for each path may lead to a misestimation of the amplitude of polarization anisotropy.

Our observations raise new questions on both the origin of continental anisotropy, and on our ability to determine its nature with current assumptions. There are many parts of the Earth where surface waves or normal mode measurements suggest large polarization anisotropy. In the Pacific ocean, $5 \%$ of polarization anisotropy has been found [26] and up to $8 \%$ of polarization anisotropy was obtained for an average model beneath Spain [23]. At global scale, $10 \%$ of anisotropy has been found in the shallow layers of the upper mantle [5] and 5\% of anisotropy is present in the upper layers of PREM [27]. All these models are averaged over wavelengths much larger than in this study and are not incompatible with locally stronger anisotropy.

Finally, whether or not the true amplitude of polarization anisotropy is determined accurately with the current assumptions, there is to our knowledge no reason to think that the reliability of its location in depth, as determined from surface wave dispersion, should be questioned. The observation of a Love/Rayleigh discrepancy beneath Australia with SH faster than SV is thus a strong independent argument that supports the presence of azimuthal anisotropy in the uppermost 200-250 km of the mantle [13] and therefore supports our two-layered anisotropic model.

\section{Acknowledgements}

We thank the IRIS and Géoscope teams for providing seismological data to supplement portable broad-band data collected by the Australian National University. J. Gaherty and S. van der Lee provided constructive reviews that helped to improve the manuscript. The figures have been made with the GMT software.[R V]

\section{References}

[1] K. Aki, K. Kanimura, Phase velocity of Love waves in Japan (part 1): Love waves from the Aleutian shock of march 1957, Bull. Earthq. Res. Inst. 41 (1963) 243-259. 
[2] T.V. McEvilly, Central u.s. crust upper-mantle structure from Love and Rayleigh wave phase velocity inversion, Bull. Seismol. Soc. Am. 54 (1964) 1997-2016.

[3] D. Mainprice, P.G. Silver, Interpretation of $S K S$-waves using samples from the subcontinental lithosphere, Phys. Earth Planet. Inter. 78 (1993) 257-280.

[4] J.P. Montagner, H.C. Nataf, A simple method for inverting the azimuthal anisotropy of surface waves, J. Geophys. Res. 91 (1986) 511-520.

[5] H.C. Nataf, I. Nakanishi, D.L. Anderson, Anisotropy and shear-velocity heterogeneities in the upper mantle, Geophys. Res. Lett. 11 (1984) 109-112.

[6] J.P. Montagner, T. Tanimoto, Global upper mantle tomography of seismic velocities and anisotropies, J. Geophys. Res. 96 (1991) 20337-20351.

[7] J.B. Gaherty, T.H. Jordan, Lehmann discontinuity as the base of anisotropic layer beneath continents, Science 268 (1995) 1468-1471.

[8] R.D. van der Hilst, B.L.N. Kennett, D. Christie, J. Grant, Skippy: Mobile broad-band arrays to study the seismic structure of the lithosphere and mantle beneath Australia, EOS Trans. Am. Geophys. Union 75 (1994) 177-181.

[9] M. Cara, J.J. Lévêque, Waveform inversion using secondary observables, Geophys. Res. Lett. 14 (1987) 1046-1049.

[10] E. Debayle, J.J. Lévêque, Upper mantle heterogeneities in the Indian Ocean from waveform inversion, Geophys. Res. Lett. 24 (1997) 245-248.

[11] E. Debayle, SV-wave azimuthal anisotropy in the Australian upper-mantle: preliminary results from automated Rayleigh waveform inversion, Geophys. J. Int. 137 (1999) 747-754.

[12] J.P. Montagner, Regional three-dimensional structures using long-period surface waves, Ann. Geophys. 4 (1986) 283-294.

[13] E. Debayle, B.L.N. Kennett, The Australian continental upper mantle: structure and deformation inferred from surface waves, J. Geophys. Res. 105 (11) (2000) 2542325450.

[14] J.J. Lévêque, E. Debayle, V. Maupin, Anisotropy in the Indian Ocean upper mantle from Rayleigh- and Love- waveform inversion, Geophys. J. Int. 133 (1998) 529540.

[15] J.J. Lévêque, M. Cara, Inversion of multimode surface wave data: evidence for sub-lithospheric anisotropy, Geophys. J. R. Astron. Soc. 83 (1985) 753-773.

[16] H. Takeuchi, M. Saito, Seismic surface waves, in: B.A. Bolt (Ed.), Methods in Computational Physics, Vol. 11, Academic Press, New York, 1972, pp. 217-295.

[17] C.E. Nishimura, D.W. Forsyth, The anisotropic structure of the upper mantle in the Pacific, Geophys. J. 96 (1989) 203-229.

[18] H.C. Nataf, Y. Ricard, 3SMAC: an a priori tomographic model of the upper mantle based on geophysical modeling, Phys. Earth Planet. Inter. 95 (1995) 101-122.

[19] C. Tong, O. Gudmundsson, B.L.N. Kennett, Shear wave splitting in refracted waves returned from the upper mantle transition zone beneath northern Australia, J. Geophys. Res. 99 (1994) 15783-15797.

[20] L.H. Estey, B.J. Douglas, Upper mantle anisotropy: A preliminary model, J. Geophys. Res. 91 (1986) 11393 11406.

[21] J.P. Montagner, Can seismology tell us anything about convection in the mantle?, Rev. Geophys. 32 (1994) 115-137.

[22] L.P. Vinnik, L.I. Makeyeva, A. Milev, A.Yu. Usenko, Global patterns of azimuthal anisotropy and deformations in the continental mantle, Geophys. J. Int. 111 (1992) 433-447.

[23] V. Maupin, M. Cara, Love-Rayleigh wave incompatibility and possible deep upper mantle anisotropy in the Iberian peninsula, Pure Appl. Geophys. 138 (1992) 429-444.

[24] A. Levshin, L. Ratnikova, Apparent anisotropy in inhomogeneous media, Geophys. J. R. Astron. Soc. 76 (1984) 65-69.

[25] V. Babuška, M. Cara, Seismic Anisotropy in the Earth, Kluwer Acad., Norwell, MA, 1991, p. 72.

[26] G. Ekström, A.M. Dziewonski, The unique anisotropy of the Pacific upper mantle, Nature 394 (1998) 168-172.

[27] A.M. Dziewonski, D.L. Anderson, Preliminary reference earth model, Phys. Earth Planet. Inter. 25 (1981) 297-356. 